\title{
Orthogonal rational functions and continued fractions
}

\author{
Adhemar Bultheel ${ }^{\mathrm{a}, *}$ Pablo González Vera ${ }^{\mathrm{b}, * *}$ Erik Hendriksen $^{\mathrm{c}}$ Olav Njåstad $^{\mathrm{d}}$ \\ ${ }^{a}$ Department of Computer Science, K.U.Leuven, Belgium \\ E-mail: Adhemar.Bultheel@cs.kuleuven.ac.be \\ ${ }^{b}$ Department of Mathematical Analysis, University La Laguna, Tenerife, Spain \\ E-mail: pglez@ull.es \\ ${ }^{c}$ Department of Mathematics, University of Amsterdam, The Netherlands. \\ E-mail: erik@wins.uva.nl \\ ${ }^{d}$ Department of Math. Sc., Norwegian Univ. of Science and Technology, Trondheim, Norway \\ E-mail: njastad@math.ntnu.no
}

\begin{abstract}
A class of continued fractions is discussed that generalize the real $J$-fractions, and which have the same relationship to orthogonal rational functions, multipoint Padé approximants and rational moment problems as real $J$-fractions have to orthogonal polynomials, onepoint Padé approximants and classical moment problems.

The setting is as follows. A sequence $\left\{\alpha_{n}\right\}$ of interpolation points in $\hat{\mathbb{R}} \backslash\{0\}$ is given. The space $\mathcal{L}$ consists of all rational functions of the form $p(z) / \omega_{n}(z)$ for some $n$ where $p(z)$ is a polynomial of degree at most $n$ and $\omega_{n}(z)=\left(1-z / \alpha_{1}\right)\left(1-z / \alpha_{2}\right) \cdots\left(1-z / \alpha_{n}\right)$. A positive linear functional $M$ on the product space $\mathcal{L} \cdot \mathcal{L}$ is given and defines an inner product on $\mathcal{L}$. A positive measure $\mu$ on $\mathbb{R}$ is a solution of the moment problem on $\mathcal{L}\left(\right.$ on $\mathcal{L} \cdot \mathcal{L}$ ) if $\int_{-\infty}^{\infty} R(t) \mathrm{d} \mu(t)=M[R]$ for all $R$ in $\mathcal{L}$ (for all $R$ in $\mathcal{L} \cdot \mathcal{L}$ ). The inner product on $\mathcal{L}$, defines an orthogonal sequence $\left\{\varphi_{n}(z)\right\}$ of functions associated with the basis $\left\{z^{n} / \omega_{n}(z)\right\}$. The sequence $\left\{\varphi_{n}(z)\right\}$ satisfies a three-term recurrence relation associated with a continued fraction. The approximants $\sigma_{n}(z) / \varphi_{n}(z)$ of that continued fraction are multipoint Padé approximants to the Stieltjes transform $F(z, \mu)=\int_{-\infty}^{\infty}(z-t)^{-1} \mathrm{~d} \mu(t)$ of solutions $\mu$ of the moment problem on $\mathcal{L} \cdot \mathcal{L}$. Like in the case of $J$-fractions, a theory of nested disks can be developed.
\end{abstract}

Keywords: orthogonal rational functions, multipoint Padé approximant, rational moment problem, continued fraction

AMS Subject classification: $42 \mathrm{C} 05,30 \mathrm{~B} 70,42 \mathrm{~A} 70,42 \mathrm{~B} 10$

\section{Introduction}

It is well known that there is a close relationship between orthogonal polynomials, special continued fractions, Padé approximants and the classical moment problems. This relationship can very briefly be described as follows.

Let a positive linear functional $M$ on the space $\Pi$ of all polynomials be given. (Equivalently: A positive definite sequence $\left\{s_{n}\right\}_{n=0}^{\infty}$ may be given, and a positive linear functional is then defined by setting $M\left[t^{n}\right]=s_{n}, n=0,1,2, \ldots$ ) A solution of the (Hamburger) moment problem for $M$ is a positive measure $\mu$ on $\mathbb{R}$ with infinite support such that $\int_{\mathbb{R}} P(t) \mathrm{d} \mu(t)=M[P]$ for all $P \in \Pi$. The functional $M$ gives rise to an inner product $\langle\cdot, \cdot\rangle$ on $\Pi$ through the formula $\langle P, Q\rangle=M[P \cdot \bar{Q}]$.

\footnotetext{
* This work is partially supported by the Belgian Program on Interuniversity Poles of Attraction, initiated by the Belgian State, Prime Minister's Office for Science, Technology and Culture. The scientific responsibility rests with the authors. ** The work of this author was supported by the Scientific Research Project of the Spanish D.G.E.S. under contract PB96-1029.
} 
By orthogonalization of the (ordered) basis $\left\{1, t, t^{2}, \ldots\right\}$ an orthogonal sequence $\left\{P_{n}\right\}$ is obtained. The sequence $\left\{P_{n}\right\}$ satisfies a three-term recurrence relation of a special form, and any polynomial sequence satisfying such a recurrence formula is a sequence of orthogonal polynomials determined by a positive linear functional on $\Pi$. Associated polynomials $\left\{Q_{n}\right\}$ are defined through the formula $Q_{n}(z)=M\left[\left(P_{n}(t)-P_{n}(z)\right) /(t-z)\right]$. The quotients $Q_{n}(z) / P_{n}(z)$ are the approximants of a continued fraction of the type real $J$-fraction. These approximants are Padé approximants to the Stieltjes transform $S(z, \mu)=\int_{\mathbb{R}}(z-t)^{-1} \mathrm{~d} \mu(t)$ for any solution $\mu$ of the moment problem for $M$ which means that $Q_{n}(z) / P_{n}(z)-S(z, \mu)=O\left(z^{-(2 n+1)}\right)$ at $z=\infty$.

The continued fraction determines for each $z \in \mathbb{C} \backslash \mathbb{R}$ a sequence of linear fractional transformations which map the upper half-plane (including the real line) onto a nested sequence of (closed) disks (for a precise definition see Section 6 ). The intersections $\Delta_{\infty}(z)$ of these disks have an invariance property: $\Delta_{\infty}(z)$ is either a single point for every $z$ (the limit point case) or a proper closed disk for every $z$ (the limit circle case). The set $\{w \in \mathbb{C}: w=S(z, \mu)$ for some solution $\mu$ of the moment problem $\}$ equals $\Delta_{\infty}(z)$, and the moment problem has a unique solution if and only if the limit point case occurs.

For details of this classical theory we refer to $[1,13,16,24,30,35,37,40,41]$.

The aim of this paper is to present the basic theory of a rational extension of the polynomial situation sketched above. The interpolation (Padé approximation) at $z=\infty$ is replaced by interpolation at arbitrary points on the extended real line, the orthogonal polynomials become orthogonal rational functions, a continued fraction generalizing the real $J$-fraction is associated with the orthogonal functions, the approximants of the continued fraction are multipoint Padé approximants to Stieltjes transforms at certain tables determined by the interpolation points. Furthermore the continued fraction again determines sequences of nested disks, the intersections again have the invariance property, and the intersections are related to Stieltjes transforms of measures solving two in general different moment problems for the functional determining the orthogonal functions.

For details concerning this rational theory which will be presented here, we refer especially to [9], and also to $[3-8,17,18,26,31,34]$. In these publications are also treated problems where the interpolation points are situated outside the real axis. Such problems are not considered in this paper. Also we do not treat explicitly the (equivalent) situation of orthogonality on the unit circle, with interpolation points on or within the circle.

Other approaches to a study of orthogonality and biorthogonality of rational functions with applications to special functions have been studied by various authors, in particular Ismail, Masson, Rachmanov, Spiridonov, and Zhedanov, and can be found e.g., in $[15,20,36,38,42]$. It can be shown that by a special choice of the poles in [20] and of the poles used in this paper, the orthogonal rational functions coincide.

It should also be mentioned that orthogonality of rational functions is closely related to orthogonality of polynomials with respect to varying weights, see especially work by Lopez, Stahl, Totik and others $[14,27-29,39]$

A theory of orthogonal rational functions was initiated by Djrbashian in 1969, see the survey paper in [12] and independently by Bultheel, Dewilde and Dym, see [2,11]. A study of orthogonal Laurent polynomials (i.e., interpolation points at $z=0$ and $z=\infty$ ) and corresponding continued fractions was initiated by Jones and Thron, see [25]. For special developments in this direction we refer to the survey article [21], and also to the papers [19,22,23,33]. 


\section{Spaces of rational functions}

Let $\left\{\alpha_{n}\right\}_{n=1}^{\infty}$ be a sequence of not necessarily distinct points on $\hat{\mathbb{R}} \backslash\{0\}$ where $\hat{\mathbb{R}}$ denotes the extended real line $\mathbb{R} \cup\{\infty\}$. (For technical reasons, it is convenient to select a distinguished fixed point in $\hat{\mathbb{R}}$ which is different from all the $\alpha_{n}$, and there is no loss of generality in placing this point at the origin.) We shall consistently use the notation $\beta_{n}=\alpha_{n}^{-1}$.

We define

$$
u_{0}=1, \quad u_{n}(z)=\prod_{k=1}^{n} \frac{z}{1-\beta_{k} z}, \quad \text { for } \mathrm{n}=1,2, \ldots
$$

and set

$$
\omega_{0}=1, \quad \omega_{n}(z)=\prod_{k=1}^{n}\left(1-\beta_{k} z\right), \quad \text { for } \mathrm{n}=1,2, \ldots
$$

Thus we may write

$$
u_{n}(z)=\frac{z^{n}}{\omega_{n}(z)}, \quad \text { for } \mathrm{n}=1,2, \ldots
$$

The linear spaces $\mathcal{L}_{n}$ and $\mathcal{L}$ are defined by

$$
\mathcal{L}_{n}=\operatorname{span}\left\{u_{0}, u_{1}, \ldots, u_{n}\right\}, \quad \text { for } \mathrm{n}=1,2, \ldots
$$

(the space is over the complex scalars) and

$$
\mathcal{L}=\bigcup_{n=0}^{\infty} \mathcal{L}_{n}
$$

A function $f$ belongs to $\mathcal{L}_{n}$ if and only of it is of the form

$$
f(z)=\frac{p(z)}{\omega_{n}(z)}
$$

where $p$ is a polynomial of degree at most $n$.

If $\alpha_{k}=\infty$ for all $k$, then $\omega_{n}(z)=1, u_{n}(z)=z^{n}$ for all $n$, and $\mathcal{L}_{n}$ is the space $\Pi_{n}$ of all polynomials of degree at most $n, \mathcal{L}$ is the space $\Pi$ of all polynomials.

Instead of $\left\{u_{0}, u_{1}, \ldots, u_{n}, \ldots\right\}$, we could work with other simple bases $\left\{v_{0}, v_{1}, \ldots, v_{n}, \ldots\right\}$ for $\mathcal{L}$ with the property that $\mathcal{L}_{n}=\operatorname{span}\left\{v_{0}, v_{1}, \ldots, v_{n}\right\}$ for all $n$. For example, if all the points $\alpha_{k}$ are finite, then $\left\{1,1 / \omega_{1}, \ldots, 1 / \omega_{n}, \ldots\right\}$ is such a basis, and if all the points $\alpha_{k}$ are finite and distinct, then $\left\{1,1 /\left(1-\beta_{1} z\right), \ldots, 1 /\left(1-\beta_{n} z\right), \ldots\right\}$ is such a basis.

Every function $f$ in $\mathcal{L}_{n}$ has a unique representation $f=\sum_{k=0}^{n} \lambda_{k} u_{k}$. We call $\lambda_{n}$ the leading coefficient of $f$ (with respect to the basis $\left\{u_{0}, u_{1}, \ldots, u_{n}\right\}$ ). When $\lambda_{n}=1$, the rational function $f$ is said to be monic.

We shall also deal with the spaces $\mathcal{L}_{m} \cdot \mathcal{L}_{n}$ consisting of all functions $h=f \cdot g$, with $f \in \mathcal{L}_{m}$ and $g \in \mathcal{L}_{n}$. It follows from (2.6) and the factorization theorem for polynomials that a function $h$ belongs to $\mathcal{L}_{m} \cdot \mathcal{L}_{n}$ if and only if it is of the form

$$
h(z)=\frac{r(z)}{\omega_{m}(z) \omega_{n}(z)},
$$

where $r$ is a polynomial of degree at most $m+n$. Thus $\mathcal{L}_{m} \cdot \mathcal{L}_{n}$ is a linear space of dimension $m+n+1$. We use the notation $\mathcal{L} \cdot \mathcal{L}$ for the space $\{h=f \cdot g: f \in \mathcal{L}, g \in \mathcal{L}\}=\bigcup_{m, n=0}^{\infty} \mathcal{L}_{m} \cdot \mathcal{L}_{n}$. 
Trivially $\mathcal{L} \subset \mathcal{L} \cdot \mathcal{L}$. If all the points in the sequence $\left\{\alpha_{k}\right\}$ are repeated an infinite number of times, then we have $\mathcal{L} \cdot \mathcal{L}=\mathcal{L}$. This is e.g., the case in the cyclic situation, where the sequence $\left\{\alpha_{k}\right\}$ consists of a finite number of finite points cyclically repeated. In particular, when $\alpha_{k}=\infty$ for all $n$ (the polynomial situation), we have $\mathcal{L} \cdot \mathcal{L}=\mathcal{L}=\Pi$.

\section{Moment problems}

The substar transform $h_{*}$ of the function $h$ is defined by

$$
h_{*}(z)=\overline{h(\bar{z})} .
$$

We note that $h_{*} \in \mathcal{L} \cdot \mathcal{L}$ if $h \in \mathcal{L} \cdot \mathcal{L}$, and that $h_{*}=h$ if $h$ belongs to $\mathcal{L} \cdot \mathcal{L}$ and if $h=f g$ with $f, g \in \mathcal{L}$ having real coefficients with respect to a basis $\left\{v_{0}, v_{1}, \ldots, v_{n}, \ldots\right\}$ for $\mathcal{L}$ such that $v_{k_{*}}=v_{k}$ for all $k$, so in particular if we use the basis $\left\{u_{0}, u_{1}, \ldots, u_{n}, \ldots\right\}$.

A linear functional $M$ on $\mathcal{L} \cdot \mathcal{L}$ is said to be positive if it satisfies

$$
M\left[h_{*}\right]=\overline{M[h]}, \quad \text { for all } h \in \mathcal{L} \cdot \mathcal{L}
$$

and

$$
M\left[f \cdot f_{*}\right]>0, \quad \text { for all } f \in \mathcal{L}, f \neq 0 .
$$

It follows from (3.2) that $M[h]$ is real when $h=f g$ with $f, g \in \mathcal{L}$ having real coefficients with respect to the basis $\left\{u_{0}, u_{1}, \ldots, u_{n}, \ldots\right\}$. For simplicity we shall in the following assume $M$ to be normalized such that $M[1]=1$.

A probability measure $\mu$ on $\mathbb{R}$ with infinite support is said to solve the moment problem on $\mathcal{L}$ if

$$
M[f]=\int_{\mathbb{R}} f(t) \mathrm{d} \mu(t), \quad \text { for all } f \in \mathcal{L}
$$

and to solve the moment problem on $\mathcal{L} \cdot \mathcal{L}$ if

$$
M[h]=\int_{\mathbb{R}} h(t) \mathrm{d} \mu(t), \quad \text { for all } h \in \mathcal{L} \cdot \mathcal{L} .
$$

A measure which solves the moment problem on $\mathcal{L} \cdot \mathcal{L}$ clearly also solves the moment problem on $\mathcal{L}$. In the polynomial situation $\left(\alpha_{k}=0\right.$ for all $\left.k\right)$ and more generally in the cyclic situation, the moment problems are equivalent.

The moment problem on $\mathcal{L}$ is always solvable when the linear functional $M$ is positive (see [9], and [7] for the equivalent problem on the unit circle). Clearly it is sufficient for $\mu$ to be a solution of the moment problem on $\mathcal{L}$ or on $\mathcal{L} \cdot \mathcal{L}$ that (3.4) or (3.5) is satisfied for some generating system for $\mathcal{L}$ or $\mathcal{L} \cdot \mathcal{L}$. In particular, $(3.4)$ is equivalent to

$$
M\left[u_{n}\right]=\int_{\mathbb{R}} u_{n}(t) \mathrm{d} \mu(t), \quad \text { for } n=0,1,2, \ldots
$$

and (3.5) is equivalent to

$$
M\left[u_{m} \cdot u_{n}\right]=\int_{\mathbb{R}} u_{m}(t) u_{n}(t) \mathrm{d} \mu(t), \quad \text { for } m, n=0,1,2, \ldots
$$

The constants $M\left[u_{m}\right]$ and $M\left[u_{m} \cdot u_{n}\right]$ are called moments. The moments are real numbers since $\left(u_{n}\right)_{*}=u_{n}$. 


\section{Orthogonal rational functions}

Let $M$ be a positive linear functional on $\mathcal{L} \cdot \mathcal{L}$. An inner product $\langle\cdot, \cdot\rangle$ on $\mathcal{L}$ can then be defined by the formula

$$
\langle f, g\rangle=M\left[f \cdot g_{*}\right], \quad \text { for } f, g \in \mathcal{L} .
$$

Let $\left\{\varphi_{n}\right\}_{n=0}^{\infty}$ be the orthonormal basis of $\mathcal{L}$ associated with the basis $\left\{u_{n}\right\}$ and with positive leading coefficients. Thus $\mathcal{L}_{n}=\operatorname{span}\left\{\varphi_{0}, \varphi_{1}, \ldots, \varphi_{n}\right\}$ for all $n,\left\langle\varphi_{j}, \varphi_{k}\right\rangle=\delta_{j, k}$ for all $j, k$, and $\varphi_{n}=\lambda_{n} u_{n}+$ $\lambda_{n-1} u_{n-1}+\cdots+\lambda_{0} u_{0}$ with $\lambda_{n}>0$. The function $\varphi_{n}$ may be written in the form

$$
\varphi_{n}(z)=\frac{p_{n}(z)}{\omega_{n}(z)}
$$

where $p_{n}$ is a polynomial of degree at most $n$.

For each $n$ we may define the linear functional $M_{n}$ and the (in general not positive definite) inner product $\langle\cdot, \cdot\rangle_{n}$ on the space $\Pi$ of polynomials by

$$
M_{n}[p]=M\left[p(z) /\left(1-\beta_{n} z\right) \omega_{n-1}(z)^{2}\right]
$$

and

$$
\langle p, q\rangle_{n}=M_{n}\left[p \cdot q_{*}\right] .
$$

Since $\varphi_{n}$ is orthogonal to all functions of the form $z^{m} / \omega_{n-1}(z)$ for $m=0,1, \ldots, n-1$, we see that $M_{n}\left[p_{n}(z) \cdot z^{m}\right]=0$ for $m=0,1, \ldots, n-1$. Thus the polynomial sequence $\left\{p_{n}\right\}$ is orthogonal with respect to the sequence of varying (in general not positive definite) inner products $\left\{\langle\cdot, \cdot\rangle_{n}\right\}$. For treatment of orthogonality with respect to varying measures, we refer to $[28,29,39]$.

We note that by construction we always have $p_{n}\left(\alpha_{n}\right) \neq 0$. We call $\varphi_{n}$ regular if $p_{n}\left(\alpha_{n-1}\right) \neq 0$, completely regular if $p_{n}\left(\alpha_{k}\right) \neq 0$ for $k=1,2, \ldots, n-1$. We say that the sequence $\left\{\varphi_{n}\right\}$ is regular (completely regular) if all $\varphi_{n}$ are regular (completely regular).

A regular sequence $\left\{\varphi_{n}\right\}$ satisfies a three-term recurrence relation. For a proof of the following theorem see [9] and [17] (the last for the situation that a finite number of finite interpolation points are cyclically repeated). See also the treatment of the equivalent situation concerning orthogonality on the unit circle in [3].

Theorem 4.1. Assume that the sequence $\left\{\varphi_{n}\right\}$ is regular. Then $\left\{\varphi_{n}\right\}$ satisfies a three-term recurrence relation of the form

$$
\varphi_{n}(z)=\left(E_{n} \frac{z}{1-\beta_{n} z}+B_{n} \frac{1-\beta_{n-1} z}{1-\beta_{n} z}\right) \varphi_{n-1}(z)-\frac{E_{n}}{E_{n-1}} \frac{1-\beta_{n-2} z}{1-\beta_{n} z} \varphi_{n-2}(z), \quad n=1,2, \ldots
$$

where $B_{n}$ and $E_{n}$ are real constants,

$$
E_{n} \neq 0, \quad \text { for all } n
$$

and by convention

$$
\left.\beta_{0}=0, \beta_{-1}=0, \quad \text { (i.e., } \alpha_{0}=\infty, \quad \alpha_{-1}=\infty\right), \quad \varphi_{-1}=0 .
$$

Recall that $\varphi_{0}=1$. Also note that $E_{0}$ can be arbitrarily chosen, since $\varphi_{-1}=0$.

We define a new orthogonal sequence $\left\{\Phi_{n}\right\}_{n=0}^{\infty}$ by the normalization

$$
\Phi_{0}=\varphi_{0}, \quad \Phi_{n}(z)=\left(E_{1} E_{2} \cdots E_{n}\right)^{-1} \varphi_{n}(z), \quad \text { for } n=1,2, \ldots
$$


The recurrence relation of Theorem 4.1 can then be written

$$
\Phi_{n}(z)=\left(\frac{z}{1-\beta_{n} z}+\frac{B_{n}}{E_{n}} \frac{1-\beta_{n-1} z}{1-\beta_{n} z}\right) \Phi_{n-1}(z)-\frac{1}{E_{n-1}^{2}} \frac{1-\beta_{n-2} z}{1-\beta_{n} z} \Phi_{n-2}(z), \quad n=1,2, \ldots
$$

with initial values

$$
\Phi_{0}=1, \quad \Phi_{-1}=0, \quad \text { and } \quad \alpha_{0}=\infty, \quad \alpha_{-1}=\infty .
$$

In the polynomial case $\left(\alpha_{k}=\infty\right.$ for all $k$ ) this recurrence relation takes the form

$$
\Phi_{n}(z)=\left(z+\frac{B_{n}}{E_{n}}\right) \Phi_{n-1}(z)-\frac{1}{E_{n-1}^{2}} \Phi_{n-2}(z), \quad n=1,2, \ldots
$$

We observe that the polynomials $\Phi_{n}$ are monic and that (4.11) is the classical recurrence relation for monic orthogonal polynomials.

A proof of the following Favard-type theorem can be found in [9]. See also [6] where the equivalent unit circle situation is treated.

Theorem 4.2. Let sequences $\left\{B_{n}\right\}_{n=1}^{\infty}$ and $\left\{E_{n}\right\}_{n=0}^{\infty}$ of real constants be given such that (4.6) is satisfied, and let the functions $\left\{\varphi_{n}\right\}$ be defined recursively by $\varphi_{0}=1$ and by (4.5), (4.7) for $n=1,2, \ldots$ Then $\varphi_{n} \in \mathcal{L}_{n} \backslash \mathcal{L}_{n-1}$ for $n=1,2, \ldots, \varphi_{n}$ is regular for all $n$, and there exists a positive linear functional $M$ on $\mathcal{L} \cdot \mathcal{L}$ such that the sequence $\left\{\varphi_{n}\right\}$ is orthonormal with respect to the associated inner product.

The situation here is more complicated than the classical (polynomial) situation. This is partly so because for a given recursion in $\mathcal{L}$, we need to define a functional $M$ on $\mathcal{L} \cdot \mathcal{L}$ with respect to which the sequence $\left\{\varphi_{n}\right\}$ is to be orthonormal.

\section{Continued fractions}

We define the associated orthogonal functions $\sigma_{n}$ and $\Sigma_{n}$ by

$$
\begin{aligned}
& \sigma_{n}(z)=M\left[\frac{\varphi_{n}(t)-\varphi_{n}(z)}{t-z}\right], \quad \text { for } n=0,1,2, \ldots \\
& \Sigma_{n}(z)=M\left[\frac{\Phi_{n}(t)-\Phi_{n}(z)}{t-z}\right], \quad \text { for } n=0,1,2, \ldots,
\end{aligned}
$$

(where $M$ operates on its argument as a function of $t$ ). Note that we may write

$$
\sigma_{n}(z)=\frac{q_{n}(z)}{\omega_{n}(z)}
$$

where $q_{n}$ is a polynomial of degree at most $n-1$. Clearly

$$
\sigma_{0}=\Sigma_{0}=0, \quad \Sigma_{n}(z)=\left(E_{1} E_{2} \cdots E_{n}\right)^{-1} \sigma_{n}(z), \quad \text { for } n=1,2, \ldots
$$

In [9] are considered functions of the second kind defined by

$$
\psi_{0}(z)=i z, \quad \psi_{n}(z)=-i M\left[\frac{1+t z}{t-z}\left(\varphi_{n}(t)-\varphi_{n}(z)\right)\right], \quad \text { for } n=1,2, \ldots
$$

The functions $\sigma_{n}$ and $\psi_{n}$ are related through the formula

$$
\psi_{n}(z)=-i\left(1+z^{2}\right) \sigma_{n}(z)+i z \varphi_{n}(z), \quad \text { for } n=0,1,2, \ldots
$$


From [9], (5.4) and (5.6) we find that the sequence $\left\{\Sigma_{n}\right\}$ satisfies the recurrence relation

$$
\Sigma_{n}(z)=\left(\frac{z}{1-\beta_{n} z}+\frac{B_{n}}{E_{n}} \frac{1-\beta_{n-1} z}{1-\beta_{n} z}\right) \Sigma_{n-1}(z)-\frac{1}{E_{n-1}^{2}} \frac{1-\beta_{n-2} z}{1-\beta_{n} z} \Sigma_{n-2}(z), \quad n=1,2, \ldots
$$

where $B_{n}$ and $E_{n}$ for $n=1,2, \ldots$ are the coefficients appearing in Theorem 4.1, and initial values are given by

$$
\Sigma_{0}=0, \quad \Sigma_{-1}=1, \quad E_{0}=-1, \quad \text { and } \quad \alpha_{0}=\infty, \quad \alpha_{-1}=\infty .
$$

(Note that a similar recurrence is obtained in Proposition 2.1 of [10].) From this follows

Theorem 5.1. Assume that the sequence $\left\{\varphi_{n}\right\}$ is regular. Then $\Sigma_{n}(z)$ and $\Phi_{n}(z)$ are the canonical numerators and denominators of a continued fraction $\mathrm{K}_{n=1}^{\infty} \frac{a_{n}(z)}{b_{n}(z)}$, where

$$
\begin{gathered}
a_{n}(z)=-\frac{1}{E_{n-1}^{2}} \frac{1-\beta_{n-2} z}{1-\beta_{n} z}, \quad \text { for } n=1,2, \ldots, \\
b_{n}(z)=\frac{z}{1-\beta_{n} z}+\frac{B_{n}}{E_{n}} \frac{1-\beta_{n-1} z}{1-\beta_{n} z}, \quad \text { for } n=1,2, \ldots
\end{gathered}
$$

We shall call a continued fraction of this form a Multipoint Padé continued fraction or an MP-fraction. A motivation for this terminology is given in Theorem 5.2. Clearly $\sigma_{n}(z)$ and $\varphi_{n}(z)$ are the canonical numerators and denominators of a continued fraction $\mathrm{K}_{n=1}^{\infty} \frac{c_{n}(z)}{d_{n}(z)}$ equivalent to $\mathrm{K}_{n=1}^{\infty} \frac{a_{n}(z)}{b_{n}(z)}$, with elements

$$
\begin{gathered}
c_{n}(z)=-\frac{E_{n}}{E_{n-1}} \frac{1-\beta_{n-2} z}{1-\beta_{n} z}, \quad \text { for } n=1,2, \ldots, \\
d_{n}(z)=E_{n} \frac{z}{1-\beta_{n} z}+B_{n} \frac{1-\beta_{n-1} z}{1-\beta_{n} z}, \quad \text { for } n=1,2, \ldots
\end{gathered}
$$

We also note that in the polynomial situation $\left(\alpha_{k}=\infty\right.$ for all $\left.k\right)$ we have

$$
a_{n}(z)=-E_{n-1}^{-2}, \quad b_{n}(z)=z+E_{n}^{-1} B_{n}, \quad \text { for } n=1,2, \ldots,
$$

and the MP-fraction thus becomes the real $J$-fraction associated with the classical Hamburger moment problem.

We shall here define the Stieltjes transform $S(z, \mu)$ of a finite measure $\mu$ on $\mathbb{R}$ by the formula

$$
S(z, \mu)=\int_{\mathbb{R}} \frac{\mathrm{d} \mu(t)}{z-t} .
$$

In [9] the Nevanlinna transform

$$
\Omega_{\mu}(z)=i \int_{\mathbb{R}} \frac{1+t z}{z-t} \mathrm{~d} \mu(t)
$$

is worked with. The two transforms are related through the formula

$$
\Omega_{\mu}(z)=i\left(1+z^{2}\right) S(z, \mu)-i z .
$$

We shall consider interpolation tables of the form $\left\{\infty, \alpha_{1}, \alpha_{1}, \ldots, \alpha_{n-1}, \alpha_{n-1}, \alpha_{n}\right\}$. We denote by $\alpha^{\#}$ the multiplicity of $\alpha$ as an entry in the table in question. By the limit at a point $\alpha$ of the table, we shall mean the one-sided limit in the upper half-plane along the normal to the real axis at the point $\alpha$, when $\alpha \neq \infty$ and along the imaginary axis when $\alpha=\infty$.

The following result can be deduced from $[8,9]$. 
Theorem 5.2. Let the positive linear functional $M$ on $\mathcal{L} \cdot \mathcal{L}$ give rise to a completely regular sequence $\left\{\varphi_{n}\right\}$ and let $\mu$ be an arbitrary solution of the moment problem on $\mathcal{L} \cdot \mathcal{L}$. Then the approximants $\sigma_{n}(z) / \varphi_{n}(z)=\Sigma_{n}(z) / \Phi_{n}(z)$ of the $M P$-fraction determined by $M$ are $[n-1 / n]$ multipoint Padé approximants to $S(z, \mu)$ at the table $\left\{\infty, \alpha_{1}, \alpha_{1}, \ldots, \alpha_{n-1}, \alpha_{n-1}, \alpha_{n}\right\}$ in the following sense:

$$
\lim _{z \rightarrow \alpha}\left[\frac{\sigma_{n}(z)}{\varphi_{n}(z)}-S(z, \mu)\right]^{(k)}=0, \quad \text { for } k=0,1, \ldots, \alpha^{\#}-1
$$

where $\alpha \in\left\{\alpha_{1}, \alpha_{1}, \ldots, \alpha_{n-1}, \alpha_{n-1}, \alpha_{n}\right\}, \alpha \neq \infty$ and

$$
\lim _{z \rightarrow \infty}\left[\frac{\sigma_{n}(z)}{\varphi_{n}(z)}-S(z, \mu)\right] z^{\infty \#}=0 .
$$

In the polynomial case $\left(\alpha_{n}=\infty\right.$ for all $\left.n\right)$ the interpolation property (5.17)-(5.18) of Theorem 5.2 takes the form

$$
\lim _{z \rightarrow \infty}\left[\frac{\sigma_{n}(z)}{\varphi_{n}(z)}-S(z, \mu)\right] z^{2 n}=0 .
$$

In this case the slightly more general property holds:

$$
\frac{\sigma_{n}(z)}{\varphi_{n}(z)}-S(z, \mu)=O\left(1 / z^{2 n+1}\right) \quad \text { as } z \rightarrow \infty .
$$

See e.g., [1]. In the rational case when $\mathcal{L} \cdot \mathcal{L}=\mathcal{L}$, a similar strengthening of the limiting properties (5.17)-(5.18) is true at all the interpolation points. (See [4].)

\section{Modified approximants and nested disks}

In all of this section, we assume that the sequence $\left\{\varphi_{n}\right\}$ determined by the functional $M$ is regular.

We define the linear fractional transformations $w \rightarrow t_{n}(z, w)$ and $w \rightarrow T_{n}(z, w)$ by

$$
\begin{gathered}
t_{0}(z, w)=w, \quad t_{n}(z, w)=\frac{a_{n}(z)}{b_{n}(z)+w}, \quad \text { for } n=1,2, \ldots, \\
T_{0}(z, w)=w, \quad T_{n}(z, w)=T_{n-1}\left(z, t_{n}(z, w)\right), \quad \text { for } n=1,2, \ldots,
\end{gathered}
$$

where $a_{n}(z)$ and $b_{n}(z)$ are as defined in (5.9)-(5.10). (Recall that by convention $\alpha_{0}=\alpha_{-1}=\infty$.) Then $T_{n}(z, 0)=\Sigma_{n}(z) / \Phi_{n}(z)=\sigma_{n}(z) / \varphi_{n}(z)$ and in general

$$
T_{n}(z, w)=\frac{\Sigma_{n}(z)+w \Sigma_{n-1}(z)}{\Phi_{n}(z)+w \Phi_{n-1}(z)}=\frac{\sigma_{n}(z)+w E_{n} \sigma_{n-1}(z)}{\varphi_{n}(z)+w E_{n} \varphi_{n-1}(z)}, \quad n=0,1,2, \ldots
$$

See e.g., $[24,30,35]$. We shall call the expressions $T_{n}(z, w)$ modified approximants of the MP-fraction $\mathrm{K}_{n=1}^{\infty} \frac{a_{n}(z)}{b_{n}(z)}$ when $w$ is of the form $w=\left[\left(1-\beta_{n-1} z\right) /\left(1-\beta_{n} z\right)\right] \tau, \tau \in \hat{\mathbb{R}}$. Thus modified approximants $R_{n}(z, \tau)$ are the expressions

$$
R_{n}(z, \tau)=T_{n}\left(z, \tau \frac{1-\beta_{n-1} z}{1-\beta_{n} z}\right)=\frac{\Sigma_{n}(z)+\tau\left(1-\beta_{n-1} z\right)\left(1-\beta_{n} z\right)^{-1} \Sigma_{n-1}(z)}{\Phi_{n}(z)+\tau\left(1-\beta_{n-1} z\right)\left(1-\beta_{n} z\right)^{-1} \Phi_{n-1}(z)},
$$

with $\tau \in \hat{\mathbb{R}}$. We note that in the polynomial case the concept of modified approximants reduces to the traditional concept of modified approximants to continued fractions. 
We shall consider mapping properties of the transformations $w \rightarrow t_{n}(z, w)$ and $w \rightarrow T_{n}(z, w)$. Let $z$ be a fixed point in the upper half-plane $\mathbb{U}=\{z: \operatorname{Im} z>0\}$. We set $\theta_{n}=\arg \left(1-\beta_{n} z\right.$ ) (note that then $\theta_{0}=0$ ) and define

$$
\begin{gathered}
\Omega_{0}=\overline{\mathbb{U}}=\{w \in \mathbb{C}: \operatorname{Im} w \geq 0\} \\
\Omega_{n}=\left\{w \in \mathbb{C}: \theta_{n-1}-\theta_{n} \leq \arg w \leq \pi+\theta_{n-1}-\theta_{n}\right\}, \quad \text { for } n=1,2, \ldots .
\end{gathered}
$$

With $\operatorname{Im} z>0$ we have $\theta_{n}<\arg z<\theta_{n}+\pi$ for all $n$. It follows that $W=z\left(1-\beta_{n} z\right)^{-1}+$ $B_{n} E_{n}^{-1}\left(1-\beta_{n-1} z\right)\left(1-\beta_{n} z\right)^{-1}+w \in \Omega_{n}$ when $w \in \Omega_{n}$. Hence $-E_{n}^{-2}\left(1-\beta_{n-2} z\right)\left(1-\beta_{n} z\right)^{-1} W^{-1} \in \Omega_{n-1}$ when $w \in \Omega_{n}$. Thus $w \in \Omega_{n}$ implies $a_{n}(z) /\left[b_{n}(z)+w\right] \in \Omega_{n-1}$. This means that for $n=1,2, \ldots$ we have

$$
t_{n}\left(z, \Omega_{n}\right) \subset \Omega_{n-1} .
$$

(Cf. the analogous argument in [18].)

For each $z$ in the open upper half-plane, $\mathbb{U}$, we set $\Delta_{n}(z)=T_{n}\left(z, \Omega_{n}\right)$. Clearly $\Delta_{n}(z)$ is a closed half-plane or a closed disk or a closed exterior of a disk. It follows from the definition that $b_{1}(z)+w$ vanishes at a point outside $\Omega_{1}$ and hence $t_{1}\left(z, \Omega_{1}\right)$ is a bounded set. Formula (6.7) gives $t_{1}\left(z, \Omega_{1}\right) \subset \Omega_{0}=\overline{\mathbb{U}}$. Again by using (6.7) we conclude that $\Delta_{n}(z)=T_{n}\left(z, \Omega_{n}\right)=T_{n-1}\left(z, t_{n}\left(z, \Omega_{n}\right)\right) \subset$ $T_{n-1}\left(z, \Omega_{n-1}\right)=\Delta_{n-1}(z)$ for $n=2,3, \ldots$. Thus $\Delta_{n}(z)$ must be a closed disk contained in $\Omega_{0}$. Consequently the following result holds:

Theorem 6.1. For $n=1,2, \ldots$ we have:

A. $\Delta_{n}(z)$ is a closed disk contained in the closed upper half-plane $\overline{\mathbb{U}}$,

B. $\Delta_{n}(z) \subset \Delta_{n-1}(z)$.

The transformation $\tau \rightarrow \tau\left(1-\beta_{n-1} z\right) /\left(1-\beta_{n} z\right)$ maps $\overline{\mathbb{U}}$ onto $\Omega_{n}$. Hence we find that the modified approximants $R_{n}(z, \tau)$ map the closed upper half-plane $\overline{\mathbb{U}}$ onto $\Delta_{n}(z)$ and the extended line $\hat{\mathbb{R}}$ onto the circle periphery $\partial \Delta_{n}(z)$.

By solving the equation $w=R_{n}(z, \tau)$ with respect to $\tau$ and setting $\operatorname{Im} \tau=0$ we find that the equation for the circle $\partial \Delta_{n}(z)$ may be written

$$
\begin{aligned}
\left(1-\beta_{n} z\right)\left(1-\beta_{n-1} \bar{z}\right) \Gamma_{n}(z, w) \overline{\Gamma_{n}(z, w)} & \\
-\left(1-\beta_{n} \bar{z}\right)\left(1-\beta_{n-1} z\right) \overline{\Gamma_{n-1}(z, w)} & \Gamma_{n-1}(z, w)=0,
\end{aligned}
$$

where

$$
\Gamma_{n}(z, w)=\sigma_{n}(z)-w \varphi_{n}(z) .
$$

(Note that here $w$ has a different meaning from that in the first part of this section.)

In [9] is proved a general Green's formula involving arbitrary solutions of the recurrence relation of the continued fraction. By replacing $w$ by $z$, and $x_{n}(z)$ and $y_{n}(z)$ by $\sigma_{n}(z)-w \varphi_{n}(z)$ in Theorem 11.4.1 of $[9]$, we get

$$
\begin{gathered}
\frac{1}{E_{n}(z-\bar{z})}\left[\left(1-\beta_{n} z\right)\left(1-\beta_{n-1} \bar{z}\right) \Gamma_{n}(z, w) \overline{\Gamma_{n-1}(z, w)}\right. \\
\left.-\left(1-\beta_{n} \bar{z}\right)\left(1-\beta_{n-1} z\right) \overline{\Gamma_{n}(z, w)} \Gamma_{n-1}(z, w)\right] \\
=\sum_{k=0}^{n-1}\left|\Gamma_{k}(z, w)\right|^{2}-\frac{w-\bar{w}}{z-\bar{z}} .
\end{gathered}
$$


Combining (6.8) and (6.10) we find that the circle $\partial \Delta_{n}(z)$ is given by

$$
\sum_{k=0}^{n-1}\left|\sigma_{k}(z)-w \varphi_{k}(z)\right|^{2}=\frac{w-\bar{w}}{z-\bar{z}}
$$

and the disk $\Delta_{n}(z)$ is given by

$$
\sum_{k=0}^{n-1}\left|\sigma_{k}(z)-w \varphi_{k}(z)\right|^{2} \leq \frac{w-\bar{w}}{z-\bar{z}}
$$

(Note that the last term in Theorem 11.4.1 of [9] has wrong sign.)

Since the sequence $\left\{\Delta_{n}(z)\right\}_{n}$ is nested, i.e., $\Delta_{n+1}(z) \subset \Delta_{n}(z)$ for all $n$, the intersection $\Delta_{\infty}(z)=$ $\bigcap_{n=1}^{\infty} \Delta_{n}(z)$ is either a single point or a proper closed disk. The following invariance result follows from [9]. See also [7] for the equivalent unit circle problem and [32] for the cyclic situation.

Theorem 6.2. The intersection $\Delta_{\infty}(z)$ consists either of a single point for every $z \in \mathbb{U}$ (the limit point case) or is a proper closed disk for every $z \in \mathbb{U}$ (the limit circle case).

The MP-fraction $\mathrm{K}_{n=1}^{\infty} \frac{a_{n}(z)}{b_{n}(z)}$ is said to converge completely for $z$ if $T_{n}\left(z, \tau\left(1-\beta_{n-1} z\right) /\left(1-\beta_{n} z\right)\right)$ converges uniformly for $\tau \in \hat{\mathbb{R}}$ to a value independent of $\tau$. (In the polynomial situation this definition coincides with the usual definition of complete convergence of real $J$-fractions, see e.g., [16].) Thus complete convergence occurs exactly in the limit point case, and the continued fraction converges completely for all or no $z$ in $\mathbb{U}$.

\section{Convergence and uniqueness}

Also in this section we shall always assume that the sequence $\left\{\varphi_{n}\right\}$ is regular.

We introduce the notation $\Sigma(z, \mathcal{L})$ and $\Sigma(z, \mathcal{L} \cdot \mathcal{L})$ for the set of Stieltjes transforms at $z$ of all solutions of the moment problem on $\mathcal{L}$ and on $\mathcal{L} \cdot \mathcal{L}$ respectively:

$$
\Sigma(z, \mathcal{L})=\{w \in \mathbb{C}: w=S(z, \mu) \text { for some solution } \mu \text { of the moment problem on } \mathcal{L}\}
$$

$\Sigma(z, \mathcal{L} \cdot \mathcal{L})=\{w \in \mathbb{C}: w=S(z, \mu)$ for some solution $\mu$ of the moment problem on $\mathcal{L} \cdot \mathcal{L}\}$

Let $n$ be fixed. By using quadrature formulas determined by the modified approximants $R_{n}(z, \tau)$ it can be shown (see [9] and [3,7] for the circle and [32] for the cyclic situation on the real line) that except for $n$ values of the real parameter $\tau$, there exists a discrete measure $\mu_{n}(\cdot, \tau)$ on $\mathbb{R}$ with the following properties: $\mu_{n}(\cdot, \tau)$ solves the truncated moment problem on $\mathcal{L}_{n-1} \cdot \mathcal{L}_{n-1}$, and $R_{n}(z, \tau)=$ $\int_{\mathbb{R}}(z-t)^{-1} \mathrm{~d} \mu_{n}(t, \tau)$. A limiting argument involving convergent subsequences of sequences $\left\{\mu_{n}\left(\cdot, \tau_{n}\right)\right\}$ leads for every point $w$ on the boundary circle $\partial \Delta_{\infty}(z)$ to a measure $\mu_{w}$ which solves the moment problem on $\mathcal{L}$ and such that $S\left(z, \mu_{w}\right)=w$. Thus $\partial \Delta_{\infty}(z) \subset \Sigma(z, \mathcal{L})$. (Note that although $\mu_{n}(\cdot, \tau)$ solves the truncated moment problem on $\mathcal{L}_{n-1} \cdot \mathcal{L}_{n-1}$, we can in general not conclude that the measures $\mu_{w}$ solve the moment problem on $\mathcal{L} \cdot \mathcal{L}$, only on $\mathcal{L}$.) The set $\Sigma(z, \mathcal{L})$ is easily seen to be convex, and hence $\Delta_{\infty}(z) \subset \Sigma(z, \mathcal{L})$.

A Hilbert space argument using Bessel's inequality analogous to the proof in the classical situation (see e.g., [1]) shows that $\Sigma(z, \mathcal{L} \cdot \mathcal{L}) \subset \Delta_{\infty}(z)$.

Thus the following result holds. 
Theorem 7.1. For every $z \in \mathbb{U}$ we have

$$
\Sigma(z, \mathcal{L} \cdot \mathcal{L}) \subset \Delta_{\infty}(z) \subset \Sigma(z, \mathcal{L})
$$

We call a moment problem determinate if it has exactly one solution, indeterminate if it has more than one solution.

All the convergent subsequences of sequences $\left\{\mu_{n}\left(\cdot, \tau_{n}\right)\right\}$ have the same solution $\mu$ as limit if and only if the continued fraction is completely convergent.

The above considerations and the results of Section 6 lead to the following theorem.

Theorem 7.2. A. If the moment problem on $\mathcal{L}$ is determinate with solution $\mu$, then the $M P$-fraction is completely convergent to $S(z, \mu)$ for all $z \in \mathbb{U}$.

$B$. If the MP-fraction is completely convergent for some $z \in \mathbb{U}$, then it is completely convergent for every $z \in \mathbb{U}$ with limit $S(z, \mu)$, where $\mu$ is a certain solution of the moment problem on $\mathcal{L}$.

$C$. If the moment problem on $\mathcal{L} \cdot \mathcal{L}$ is solvable and the MP-fraction is completely convergent for some $z \in \mathbb{U}$, then the moment problem on $\mathcal{L} \cdot \mathcal{L}$ is determinate and the MP-fraction converges completely for every $z \in \mathbb{U}$ to $S(z, \mu)$ where $\mu$ is the unique solution of the moment problem on $\mathcal{L} \cdot \mathcal{L}$.

\section{References}

[1] N.I. Akhiezer. The classical moment problem and some related questions in analysis. Hafner, New York, 1965.

[2] A. Bultheel and P. Dewilde. Orthogonal functions related to the Nevanlinna-Pick problem. In P. Dewilde, editor, Proc. 4th Int. Conf. on Math. Theory of Networks and Systems at Delft, pages 207-212, North-Hollywood, 1979. Western Periodicals.

[3] A. Bultheel, P. González-Vera, E. Hendriksen, and O. Njåstad. Orthogonal rational functions with poles on the unit circle. J. Math. Anal. Appl., 182:221-243, 1994.

[4] A. Bultheel, P. González-Vera, E. Hendriksen, and O. Njåstad. Orthogonality and boundary interpolation. In A.M. Cuyt, editor, Nonlinear Numerical Methods and Rational Approximation II, pages 37-48. Kluwer, 1994.

[5] A. Bultheel, P. González-Vera, E. Hendriksen, and O. Njåstad. Recurrence relations for orthogonal functions. In S.C. Cooper and W.J. Thron, editors, Continued Fractions and Orthogonal Functions, volume 154 of Lecture Notes in Pure and Appl. Math., pages 24-46. Marcel Dekker, 1994.

[6] A. Bultheel, P. González-Vera, E. Hendriksen, and O. Njåstad. A Favard theorem for rational functions with poles on the unit circle. East J. Approx., 3:21-37, 1997.

[7] A. Bultheel, P. González-Vera, E. Hendriksen, and O. Njåstad. A rational moment problem on the unit circle. Methods Appl. Anal., 4(3):283-310, 1997.

[8] A. Bultheel, P. González-Vera, E. Hendriksen, and O. Njåstad. Interpolation of Nevanlinna functions by rationals with poles on the real line. In W.B. Jones and A.S. Ranga, editors, Orthogonal Functions, Moment Theory and Continued Fractions: Theory and Applications, volume 199 of Lecture Notes in Pure and Applied Mathematics, pages 101-110. Marcel Dekker, 1998.

[9] A. Bultheel, P. González-Vera, E. Hendriksen, and O. Njåstad. Orthogonal rational functions, volume 5 of Cambridge Monographs on Applied and Computational Mathematics. Cambridge University Press, 1999.

[10] A. Bultheel, P. González-Vera, E. Hendriksen, and O. Njåstad. A rational Stieltjes problem. Appl. Math. Comput., 1999. To appear.

[11] P. Dewilde and H. Dym. Schur recursions, error formulas, and convergence of rational estimators for stationary stochastic sequences. IEEE Trans. Inf. Th., IT-27:446-461, 1981.

[12] M.M. Djrbashian. A survey on the theory of orthogonal systems and some open problems. In P. Nevai, editor, Orthogonal polynomials: Theory and practice, volume 294 of Series C: Mathematical and Physical Sciences, pages 135-146, Boston, 1990. NATO-ASI, Kluwer Academic Publishers.

[13] G. Freud. Orthogonal polynomials. Pergamon Press, Oxford, 1971. 
[14] A. Gonchar and G. López. On Markov's theorem for multipoint Padé approximants for functions of Stieltjes type. Math. USSR-Sb., 105:512-524, 1978. Enlish translation. Math. USSR-Sb, 34 (1978) 449-459.

[15] D.P. Gupta and D.R. Masson. Continued fractions and orthogonality. Trans. Amer. Math. Soc., 350:769-808, 1998.

[16] H. Hamburger. Ueber eine Erweiterung des Stieltjesschen Moment Problems I. Math. Ann., 81:235-319, 1920.

[17] E. Hendriksen and O. Njåstad. A Favard theorem for rational functions. J. Math. Anal. Appl., 142(2):508-520, 1989.

[18] E. Hendriksen and O. Njåstad. Positive multipoint Padé continued fractions. Proc. Edinburgh Math. Soc., 32:261$269,1989$.

[19] E. Hendriksen and H. van Rossum. Orthogonal Laurent polynomials. Proc. of the Kon. Nederl. Akad. Wetensch, Proceedings A, 89(1):17-36, 1986.

[20] M.E.H. Ismail and D.R. Masson. Generalized orthogonality and continued fractions. J. Approx. Theory, 83:1-40, 1995.

[21] W.B. Jones and O. Njåstad. Orthogonal Laurent polynomials and strong moment theory. J. Comput. Appl. Math., 105:51-91, 1999.

[22] W.B. Jones, O. Njåstad, and W.J. Thron. Continued fractions and strong Hamburger moment problems. Proc. London Math. Soc., Ser III, 47(3):363-384, 1983.

[23] W.B. Jones, O. Njåstad, and W.J. Thron. Orthogonal Laurent polynomials and the strong Hamburger moment problem. J. Math. Anal. Appl., 98:528-554, 1984.

[24] W.B. Jones and W.J. Thron. Continued Fractions. Analytic Theory and Applications. Addison-Wesley, Reading, Mass., 1980.

[25] W.B. Jones and W.J. Thron. Orthogonal Laurent polynomials and Gaussian quadrature. In K. Gustafson and W.P. Reinhardt, editors, Quantum mechanics in mathematics, chemistry and physics, pages 449-455, New York, 1984. Plenum.

[26] X. Li. Regularity of orthogonal rational functions with poles on the unit circle. J. Comput. Appl. Math., 105:371-383, 1999.

[27] G.L. Lopes [López-Lagomasino]. Conditions for convergence of multipoint Padé approximants for functions of Stieltjes type. Math. USSR-Sb., 35:363-376, 1979.

[28] G.L. Lopes [López-Lagomasino]. On the asymptotics of the ratio of orthogonal polynomials and convergence of multipoint Padé approximants. Math. USSR-Sb., 56:207-219, 1985.

[29] G.L. López [López-Lagomasino]. Asymptotics of polynomials orthogonal with respect to varying measures. Constr. Approx., 5:199-219, 1989.

[30] L. Lorentzen and H. Waadeland. Continued fractions with applications, volume 3 of Studies in Computational Mathematics. North-Holland, 1992.

[31] O. Njåstad. An extended Hamburger moment problem. Proc. Edinburgh Math. Soc., 28:167-183, 1985.

[32] O. Njåstad. Unique solvability of an extended Hamburger moment problem. J. Math. Anal. Appl., 124:502-519, 1987.

[33] O. Njåstad. Solutions of the strong Hamburger moment problem. J. Math. Anal. Appl., 197:227-248, 1996.

[34] O. Njåstad and W.J. Thron. Unique solvability of the strong Hamburger moment problem. J. Austral. Math. Soc. (Series A), 40:5-19, 1986.

[35] O. Perron. Die Lehre von den Kettenbrüchen. Teubner, 1977.

[36] M. Rahman and S. Suslov. Classical biothogonal rational functions. In Methods of Approximation Theory in Complex Analysis and Mathematical Physics IV, volume 1550 of Lecture Notes in Math., pages 131-146. Springer Verlag, 1993.

[37] J.A. Shohat and J.D. Tamarkin. The problem of moments, volume 1 of Math. Surveys. Amer. Math. Soc., Providence, R.I., 1943.

[38] W. Spiridonov and A. Zhedanov. Spectral transformation chains and some new biorthogonal rational functions. Comm. Math. Phys., 1999. To appear.

[39] H. Stahl and V. Totik. General orthogonal polynomials. Encyclopedia of Mathematics and its Applications. Cambridge University Press, 1992.

[40] T.J. Stieltjes. Recherches sur les fractions continues. Ann. Fac. Sci. Toulouse, 8:J.1-122, 9:A.1-47, 1894. English transl.: Oeuvres Complèts, Collected Papers, Springer Verlag, 1993, Vol. 2, 609-745. 
[41] G. Szegő. Orthogonal polynomials, volume 33 of Amer. Math. Soc. Colloq. Publ. Amer. Math. Soc., Providence, Rhode Island, 4th edition, 1975. First edition 1939.

[42] A. Zhedanov. Biorthogonal rational functions and generalized eigenvalue problem. J. Approx. Theory, 101(2):303$329,1999$. 